\title{
Generating Learning Data for Hierarchical Vegetation Classification Methods using Support Vector Machine
}

\author{
Masatomo SuZUKI*, Yoichi KAGEYAMA*, Chikako ISHIZAWA*, Makoto NISHIDA**, \\ Koshi SATO***, Mitsuyoshi KANEKO*** and Takashi NAGAKI ${ }^{\star \star *}$ \\ * Department of Mathematical Science and Electrical-Electronic-Computer Engineering, \\ Graduate School of Engineering Science, \\ Akita University, 1-1, Tegata Gakuen-machi, Akita-shi, Akita 010-8502, Japan \\ ** The Open University of Japan, 1-1, Tegata Gakuen-machi, Akita-shi, Akita 010-8502, Japan \\ *** Akita Office of River and National Highway, Tohoku Regional Bureau, \\ Ministry of Land, Infrastructure, Transport and Tourism \\ 1-10-29, Sanno, Akita-shi, Akita 010-0951, Japan \\ E-mail:kageyama@ie.akita-u.ac.jp
}

\begin{abstract}
In a previous study, we developed a method for classifying vegetation on a river bank managed by the Ministry of Land, Infrastructure, Transport and Tourism, by using images acquired from the Omonogawa River flowing through the Akita Prefecture. We focused specifically on color and texture information from those images, and proposed a method for classifying vegetation with a support vector machine, which is a pattern recognition model. However, the color features of the turf and the harmful vegetation, Fallopia japonica, were roughly the same when calculated during the same season across different years. Distinguishing images based on the acquired seasons should enable high-precision classification. Thus, in this study, we develop a learning data generation method that can classify new data. Specifically, we categorize the learning data by month and determine parameters for the appropriate unlearned data. An experiment is conducted using data generated from May, June, and July of 2015 and 2016. We found that the proposed generation method can classify river bank vegetation with high accuracy in comparison with the previous approach.
\end{abstract}

Keywords : Support vector machine, Learned data, Vegetation classification, River bank

\section{INTRODUCTION}

A river bank is an important facility, created to prevent running water from flowing outside the river during floods and to protect people's lives and property. The turf on the river bank's slope prevents the bank from collapsing during flooding. However, the growth of harmful vegetation may cause the turf to die, weakening the river bank. Therefore, the river banks under the management of the Akita Office of River and National Highway, Ministry of Land, Infrastructure, Transport and Tourism (MLIT), is weeded twice per year with the purposes of conserving and preserving the rivers [1]. However, judgment of the weeding timing is conducted subjectively. Management cameras are set up along the river bank; thus, it is possible to acquire still river bank images at any point in time.

In recent years, classification methods using machine learning have been developed in order to classify target vegetation with high accuracy [2] To date, we have proposed a ratio estimation algorithm for harmful vegetation to reduce the burden on river management workers [3]. In addition, we proposed a method for classifying hierarchical vegetation [4], focusing on color and texture information [5] obtained from images, using a support vector machine (SVM) [6]. The SVM is a pattern recognition model, which uses supervised learning obtained from the Omonogawa River bank imagery. To practically use this method, we must classify new point data and new obtained data of the same point. However, the growth state and color information of vegetation changes over time. Thus, it is desirable to classify using appropriate SVM parameters and learning data for each season. To improve the versatility of vegetation classification for the river bank, we examine classification accuracy with parameters and learning data using river bank images, monthly.

\section{TARGET VEGETATION}

River bank vegetation can be roughly divided into "turf" and "harmful vegetation." Turf grows fine roots in the ground and exhibits two features: reducing amounts of soil outflow; and behaving like a mesh, storing a great deal of water in the soil. These features contribute to the protection of river banks from flood waters, which is why turf is artificially laid on river banks' slopes. In fact, experimental results have reported that the river bank resists flowing water when turf is laid appropriately [7]. An example of turf on a river bank's slope is shown in Figure 1(a).

Fallopia japonica is a harmful vegetation type, which grows high and has a tough, broad leaf, resulting in sunlight being shut out, causing the death of the turf. Additionally, because its roots 


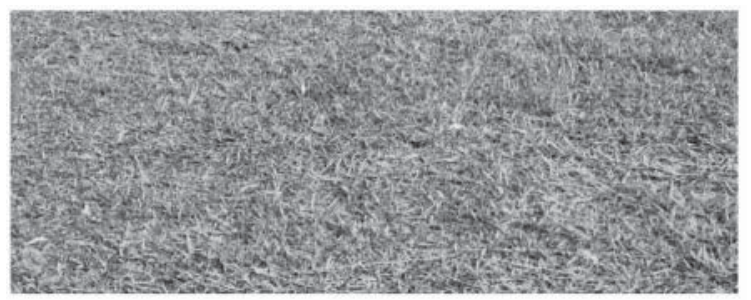

(a) Turf (acquired on May 29, 2015)

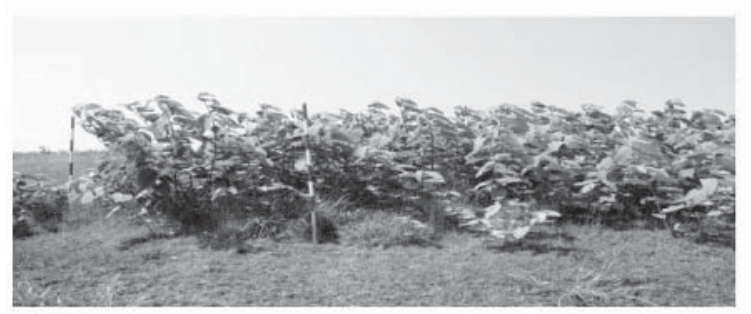

(b) Fallopia japonica (acquired on May 29, 2015)

Figure 1 Example of target vegetation

are thick and extend far in all directions, the river bank becomes hollowed out. Therefore, river bank weakening occurs. Figure 1(b) shows an example of Fallopia japonica. This study focuses on the turf and Fallopia japonica as the target vegetation.

\section{IMAGE DATA}

In this study, we use a river bank image of $720 \times 480$ pixels, and 256 gradations for each RGB, acquired by the camera equipment of a multicast CCTV image-providing system. Image data are acquired regularly by the Akita Office of River and National Highway, MLIT, approximately once a week, around 9 a.m. The distance extent is of three types: zoom 1, a high zoom; zoom 2, a normal zoom; and zoom 3, a low zoom.

A total of 35 images of zoom 3, of Fallopia japonica mixed with turf, are used. Images were acquired at two places, Furukawa purification facility and Barazima river park. Among the 21 images taken in 2015, four are taken in May; eight are taken in June; and nine are taken in July. Among the 14 taken in 2016, five are taken in May; three are taken in June; and six are taken in July. Image data acquired when there is no Fallopia japonica following weeding are excluded. An example of an acquired river bank image is shown in Figure 2. There is distortion in the left and right ends of the river bank image, owing to the camera's specification. In

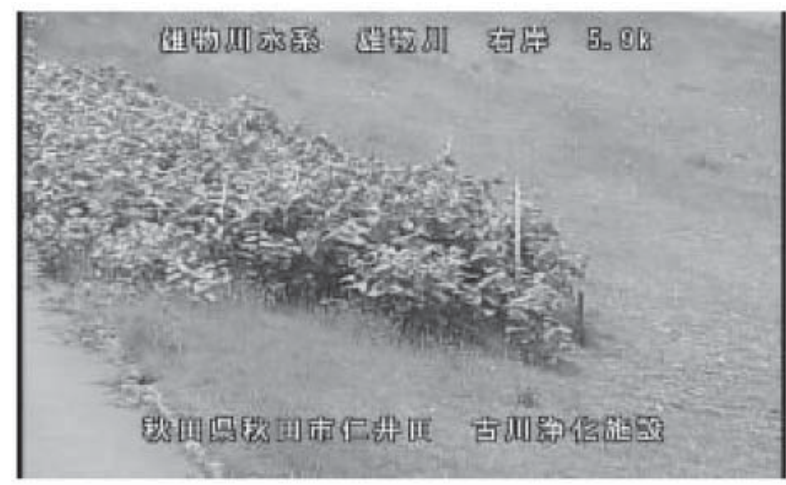

Figure 2 Example of image used Example of image used $(720 \times 480$ pixels, Furukawa Purification Facility) addition, the character strings represent the points at the top and bottom of the river bank image, respectively.

\section{METHOD FOR HIERARCHICAL VEGETATION CLASSIFICATION}

Figure 3 shows the hierarchical vegetation classification method flowchart. The classification method consists of three processes: target area extraction (i.e., (1) and (2) in Figure 3), feature value calculation (i.e., (3) and (4) in Figure 3), and hierarchical vegetation classification (i.e., (5) to (9) in Figure 3).

\subsection{Target area extraction}

The river bank image contains distortion as well as characters, as shown in Figure 2. Distortion sizes are 20 pixels to the left and right of the image. The character string area sizes are 110 pixels (Furukawa Purification Facility) and 115 pixels (Barazima River Park), at the top and bottom of the images in total, respectively. We first remove these areas. An example of the removal results is shown in Figure 4 (a). The difference in size following removal is a result of the character string size difference of the given point. Next, we extract the target area for vegetation classification based on the previous method [4]. The equation for extracting the vegetation area is as follows.

$$
\begin{aligned}
& 90^{\circ}<\theta<225^{\circ} \\
& S \geq 0.03,
\end{aligned}
$$

where $\theta$ is the metric hue angle, and $\mathrm{S}$ is the saturation [8]. The metric hue angle indicates the hue at an angle with the axis of a* in the $\mathrm{L}^{*} \mathrm{a}^{*} \mathrm{~b}^{*}$ color system. Because most of the vegetation area belongs to the second quadrant, and some to the third quadrant, pixels in the range of Eq. (1) are assumed to be vegetation areas.

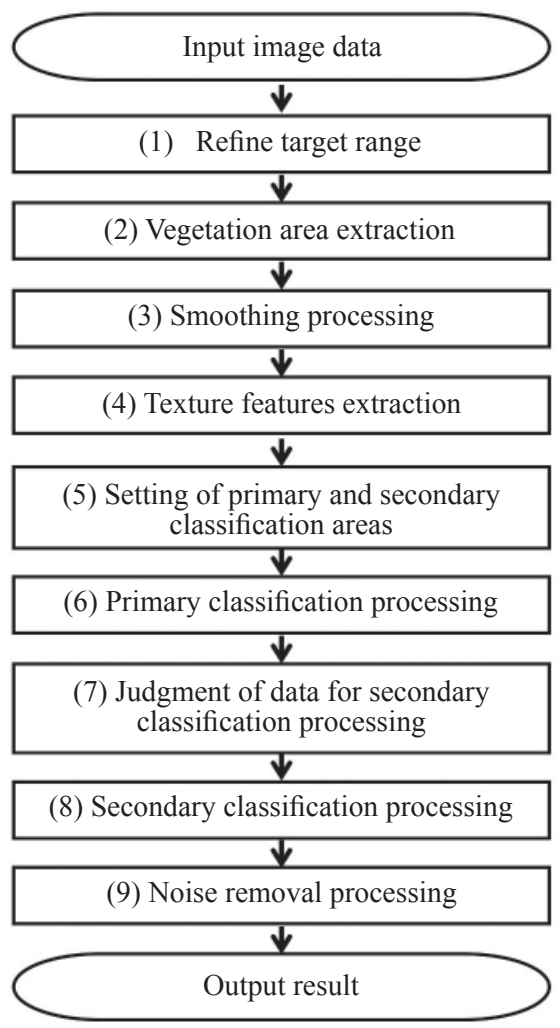

Figure 3 Flowchart of hierarchical vegetation classification method 


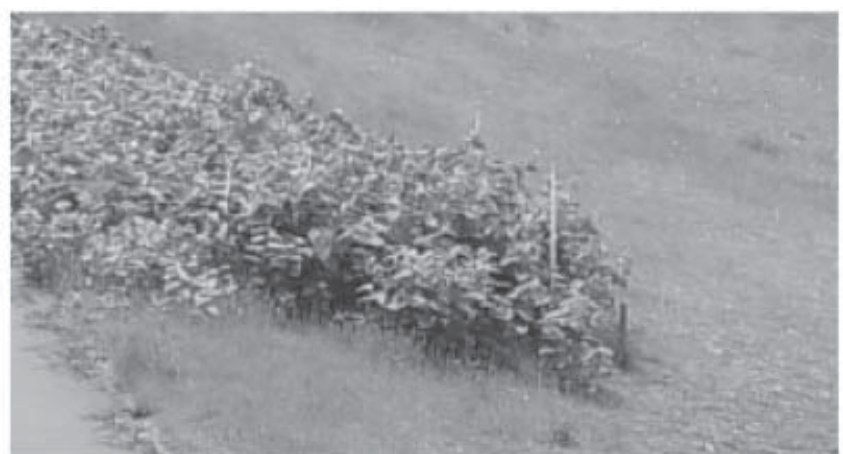

(a) Example of image resulting from distortion and character deletion

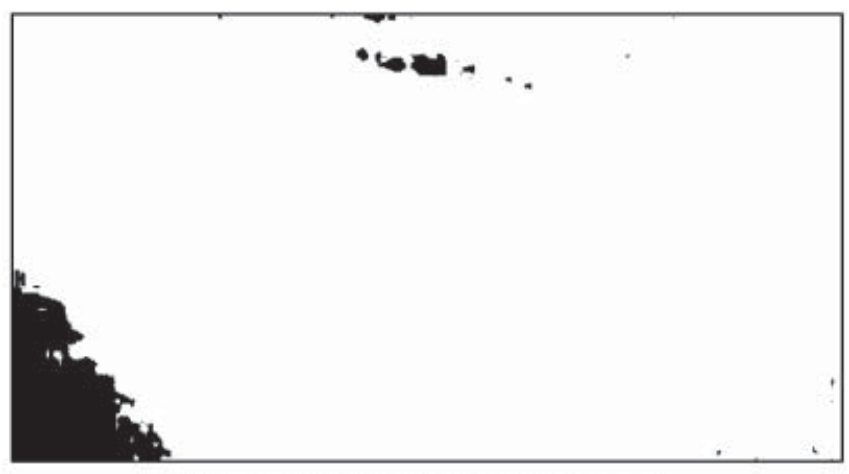

(b) Example of target area extraction result

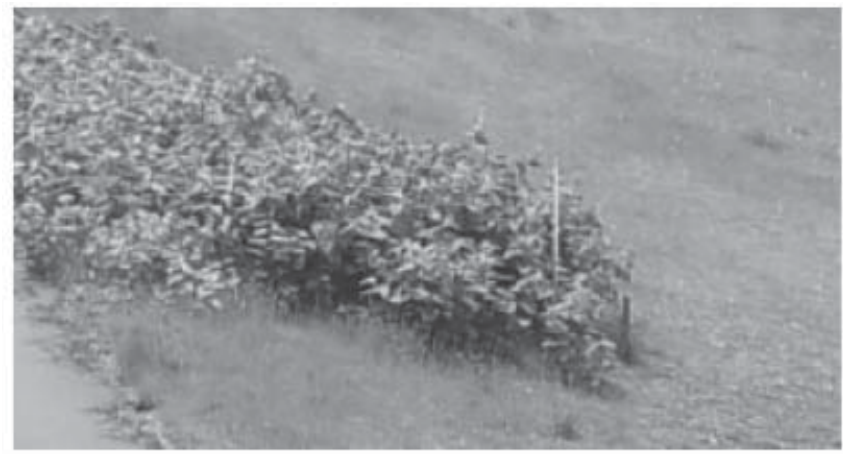

(c) Example of image after smoothing processing

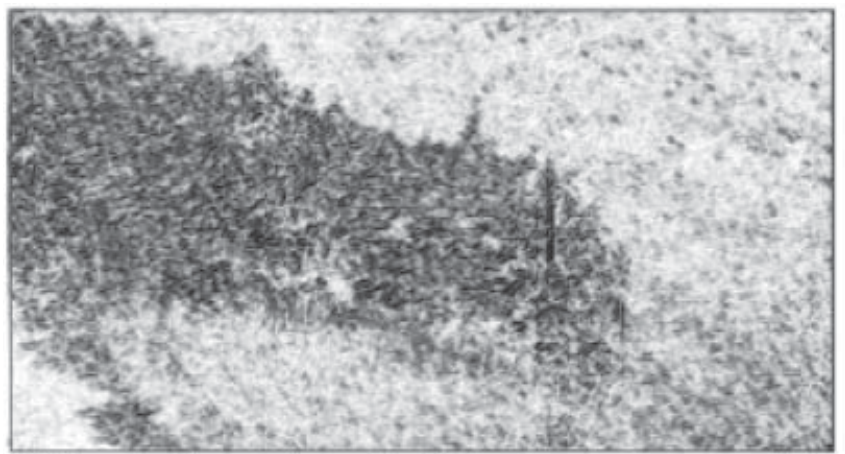

(d) Example of image of calculated feature amount "homogeneity"

Figure 4 Example of processing result

$(700 \times 370$ pixels, Furukawa Purification Facility)

Thus, they are examined in $15^{\circ}$ increments. Moreover, there are non-vegetation areas, such as roads, which are included. Owing to vegetation saturation being higher than that of structures, such as roads, it is possible to judge the pixels corresponding to the vegetation area obtained by Eq. (2). An example of the extraction result is shown in Figure 4(b), where the white pixels indicate an extracted area as a vegetation area.

\subsection{Feature value calculation}

Turf color information tends to be similar in the river bank image, whereas the color change is extensive in the Fallopia japonica area. Furthermore, depending on the acquisition condition of the river bank images, it may be difficult to capture detailed turf color changes. Texture is one of the indices for indicating surface uniformity. When applying texture, turf with a small color change has high uniformity, whereas Fallopia japonica, with a large color change, has low uniformity. Thus, it appears to be possible to distinguish the two. Therefore, we calculate the texture feature amounts per the following two steps.

First, we carry out a smoothing process for alleviating color change in the turf area. An example of the image following the smoothing process is shown in Figure 4(c). Next, a co-occurrence matrix [5] was calculated for each component of the RGB values in the smoothed image. Using the obtained co-occurrence matrix, whose processing area size is $3 \times 3$, displacement, $\delta$, is $1,45^{\circ}$, and gradations are 64 , homogeneity [5], a texture feature amount, is calculated. In this study, homogeneity, representing the uniformity of color change, is used to discriminate between turf and Fallopia japonica, as well as color information. Figure 4 (d) shows an example of the calculated homogeneity.

\subsection{Hierarchical vegetation classification}

4.3.1 SVM outline: SVM is a discrimination method that learns from reference data and performs classification using a separation plane or separation curved surface. There are two types of SVM: hard-margin and soft-margin, which we use. Soft-margin SVM allocates a penalty multiplier to misclassification, thus enabling flexible classification for error identification. For classification using a nonlinear hyperplane, a radial basis function (RBF) kernel [6] is used. In the classification method using supervised learning, feature space should be classified by linear separation. However, because the feature space used is complicated, nonlinear separation is necessary. Therefore, by appropriately setting multiple parameters at the time of learning, SVM enables classification within a complex feature space. In the SVM used for this study, it is necessary to provide $\mathrm{C}$ and $\gamma$, where $\mathrm{C}$ is a parameter for determining the margin of the soft-margin SVM, and $\gamma$ is a parameter for controlling the RBF kernel radius [6]. The above two parameters are calculated by grid search [9]. This method examines all combinations of parameters and selects the combinations with the best results.

4.3.2 Classification using SVMs: Prior learning is required to carry out vegetation classification using SVM. It is necessary to prepare classes to which pixels belong, and the feature amount is used as learning data. Therefore, based on the river bank manager's knowledge, target image data are divided by using colors. Figure 5 shows reference data derived from Figure 2.

Using the reference data and feature amount images, many pixels from each image class are randomly obtained with the learning data. During the vegetation classification process, pixels are classified by the learning data. That is, the feature amount of each pixel in the vegetation area of the classification target image is input to the SVM, and pixels are classified as turf or Fallopia japonica. A flowchart of the SVM classification is illustrated in Figure 6 . The proposed method narrows down the learning data by a histogram comparison. Therefore, classification accuracy would decrease in comparison with the previous method when the image of the histogram having features close to the image to be classified 


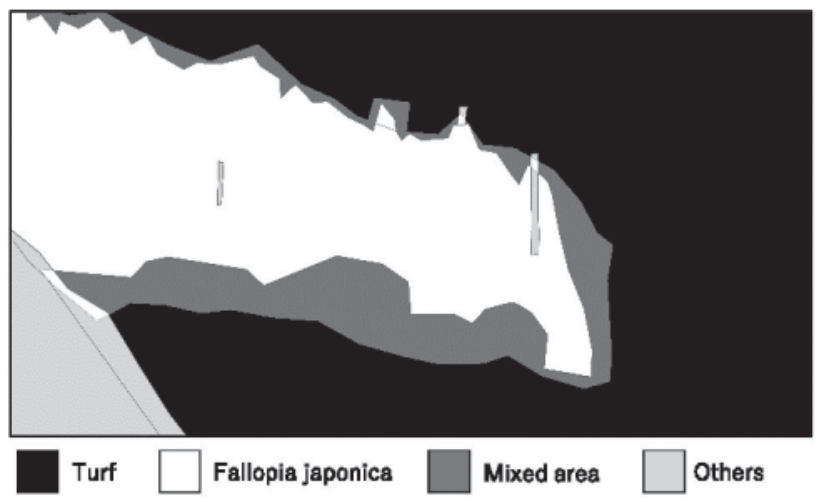

Figure 5 Example of reference data

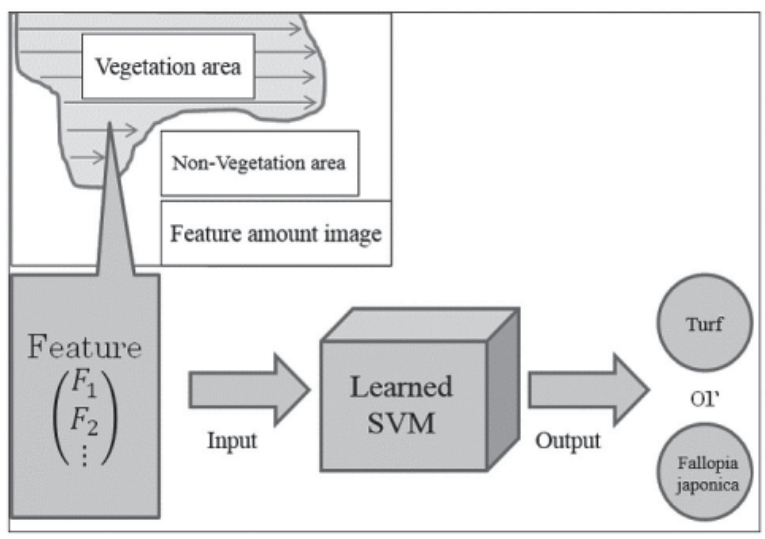

Figure 6 Flowchart of classification using SVM

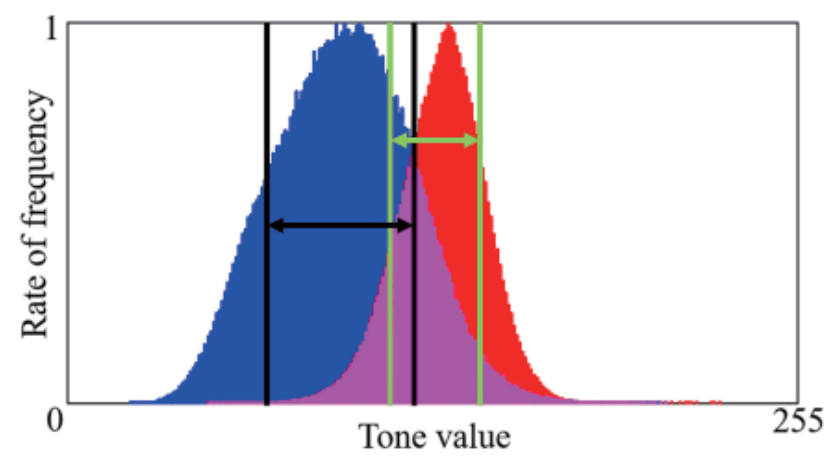

Figure 7 Example of setting feature area

does not exist in the preset data.

Step 1. Setting of primary and secondary classification areas: To perform highly accurate classification, it is necessary to separate the mixed turf and Fallopia japonica areas from the others. A range with a cumulative frequency of $68.27 \%$ is extracted as an area showing the main turf and Fallopia japonica features, based on the average values of the two in each feature amount. Figure 7 shows an example of setting the feature area. The mixed area is extracted as the secondary classification area, whereas that outside of the mixed area is extracted as the primary classification area.

Step 2. Primary classification processing: We perform learning and classification on the primary classification area with SVM, using the RGB values and homogeneity calculated from each RGB element, with a total of six features. Figure 8(a) shows an example

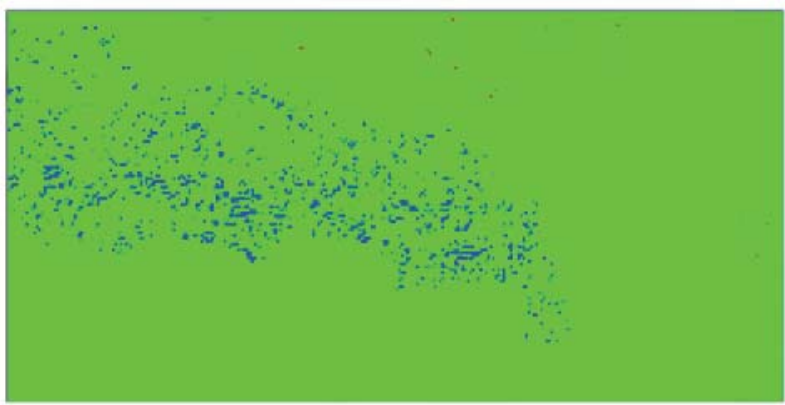

(a) Example of image result from primary classification process

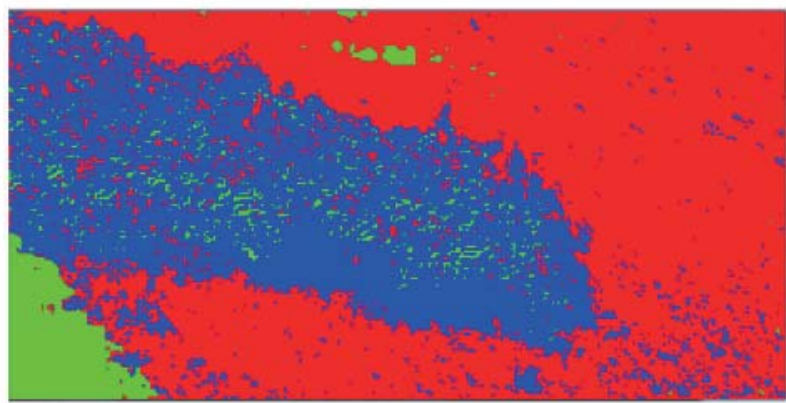

(b) Example of image result from secondary classification process

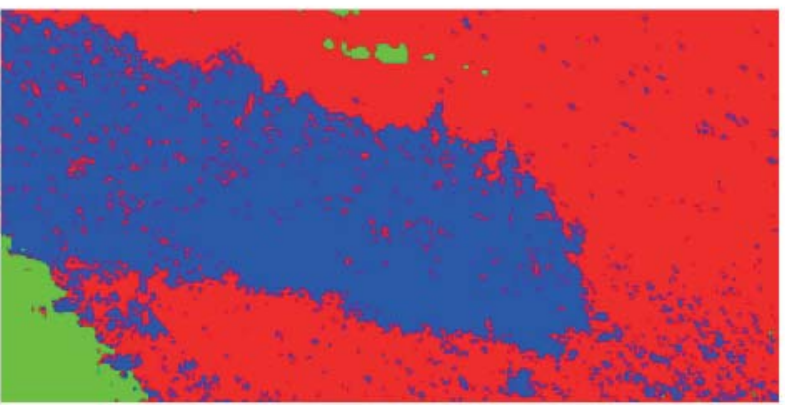

(c) Example of image from final classification result

Turf

Fallopia japonica

Others

Figure 8 Example of image of classification result

of the resulting image from the primary classification process.

Step 3. Judgment of data for secondary classification processing:

To improve the mixed area classification accuracy, it is necessary to select the learning data appropriately, per the classification target data characteristics.

Therefore, an image from the learning data set is judged as being either valid learning data or not. Specifically, we compare the histograms of images to be classified and those of the learning data, and learning data are acquired from the image with the highest similarity in the set. In this study, the Chi-square value [10] is used to compare histogram similarity. The Chi-square value becomes 0 when the histograms are perfectly matched, and the value increases as similarity decreases. In the above processing, it is possible to reduce the influence of the change in the RGB values due to the data acquisition condition.

Step 4. Secondary classification processing: Our previous study clarified that the R-value was the most suitable value for classification [4], so we adopted it for the secondary classification area. Thus, learning by SVM is performed using R-values and homogeneity, with a total of four features, and the secondary classification region pixels are reclassified. Figure 8(b) shows an example of an image result from the secondary classification 
process.

Step 5. Noise removal processing: Noise in units of several pixels generated due to sunlight irradiation conditions exists in the SVM classification results. Thus, integrated results of the primary and secondary classification subjected to noise removal by dilation and erosion processes are output as the final classification result. Fig. 8(c) shows an example image following the final classification result.

\section{EXPERIMENTAL METHOD}

To improve the versatility of the hierarchical vegetation classification method, we compare these with the previous method [4]. Furthermore, the pixel matching rate is calculated as follows.

Pixel matching rate $=\frac{\text { number of correctly classified pixels }}{\text { total number of pixels }}$

We set the 2015 data as learning data, and that of 2016 as new data, categorizing the learning data by month. We also calculate the parameters using the data of 2015, and assign these to the new data. Then, we perform evaluation by means of leave-one-out cross-validation to calculate the pixel matching rate.

\section{RESULTS AND DISCUSSION}

Table 1 lists the pixel matching rates obtained by using the hierarchical vegetation classification method, whereas Table 2 lists those obtained by means of the previous method. Compared with the previous method, the average value of the pixel matching rate improves by $5.85 \%$ at the maximum turf, Fallopia japonica, and the whole. Additionally, the classification accuracy of data on July 13, 2016 is largely improved by the hierarchical vegetation classification method (See Tables 1 and 2, red frame). This suggests that good vegetation classification is possible by learning, suitable for the growth state of vegetation. The above results suggest that the hierarchical vegetation classification method is useful for vegetation classification compared to the previous method.

\section{CONCLUSION}

In this paper, we examined the versatility of the hierarchical vegetation classification method using an SVM by means of different parameters for two acquisition condition types. The following conclusions were obtained:

1. Classification using hierarchical processing for mixed areas between turf and Fallopia japonica with RGB values and homogeneity contributes to enhanced accuracy.

2. The hierarchical vegetation classification method can improve classification accuracy for data with low classification accuracy in the conventional method; it contributes to improvement in accuracy.

\section{Acknowledgements}

The authors thank the Tohoku Regional Bureau, Ministry of Land, Infrastructure, Transport and Tourism, for their assistance in conducting the experiments.

\section{References}

[1] Akita Office of River and National Highway, Tohoku Regional Bureau, Ministry of Land, Infrastructure, Transport and Tourism Web Site: http://www.thr.mlit.go.jp/akita/index. html (accessed 17.04.28).

[2] Suzuki T.; Tsuchiya T.; Suzuki S.; Yamaba A., "Vegetation
Table 1 Pixel-matching rate obtained using hierarchical vegetation classification method

\begin{tabular}{|c|c|c|c|c|}
\hline \multirow{2}{*}{ Point } & \multirow{2}{*}{ Target data } & \multicolumn{3}{|c|}{ Hierarchical vegetation chssification method } \\
\hline & & Turf & Fallopia japonica & All areas \\
\hline \multirow{7}{*}{$\begin{array}{c}\text { Furukawa } \\
\text { Purification } \\
\text { Facility }\end{array}$} & 20160506 & 90.97 & 99.54 & 92.97 \\
\hline & 20160520 & 95.35 & 98.01 & 96.36 \\
\hline & 20160527 & 89.79 & 99.86 & 93.46 \\
\hline & 20160617 & 96.52 & 98.17 & 96.96 \\
\hline & 20160713 & 97.66 & 95.12 & 96.91 \\
\hline & 20160722 & 97.50 & 93.29 & 96.31 \\
\hline & 20160729 & 91.47 & 98.69 & 93.53 \\
\hline \multirow{7}{*}{$\begin{array}{c}\text { Barazima } \\
\text { River Park }\end{array}$} & 20160520 & 84.80 & 98.68 & 88.68 \\
\hline & 20160527 & 82.79 & 99.33 & 86.53 \\
\hline & 20160603 & 92.52 & 81.94 & 88.08 \\
\hline & 20160617 & 99.65 & 90.60 & 98.55 \\
\hline & 20160713 & 99.13 & 89.80 & 97.84 \\
\hline & 20160722 & 98.63 & 96.81 & 98.31 \\
\hline & 20160729 & 97.59 & 97.63 & 97.59 \\
\hline \multicolumn{2}{|c|}{ Average } & 93.88 & 95.53 & 94.43 \\
\hline
\end{tabular}

Table 2 Pixel-matching rate obtained using previous method

\begin{tabular}{|c|c|r|r|r|}
\hline \multirow{2}{*}{ Point } & \multirow{2}{*}{ Target data } & \multicolumn{3}{|c|}{ Previous method } \\
\cline { 3 - 5 } & & \multicolumn{1}{|c|}{ Turf } & Fallopia japonica & \multicolumn{1}{c|}{ All areas } \\
\hline \multirow{4}{*}{$\begin{array}{c}\text { Furukawa } \\
\text { Purification }\end{array}$} & 20160506 & 94.32 & 98.93 & 95.40 \\
\cline { 2 - 5 } & 20160520 & 99.29 & 95.61 & 97.89 \\
\cline { 2 - 5 } Facility & 20160527 & 97.06 & 99.85 & 98.08 \\
\cline { 2 - 5 } & 20160617 & 92.96 & 98.98 & 94.57 \\
\cline { 2 - 5 } & 20160713 & 34.32 & 99.77 & 53.70 \\
\cline { 2 - 5 } & 20160722 & 72.03 & 98.93 & 79.69 \\
\cline { 2 - 5 } & 20160729 & 67.96 & 99.61 & 77.01 \\
\hline \multirow{4}{*}{ Barazima } & 20160520 & 98.28 & 93.71 & 97.00 \\
\cline { 2 - 5 } River Park & 20160527 & 84.80 & 99.40 & 88.10 \\
\cline { 2 - 5 } & 20160603 & 97.21 & 80.40 & 90.17 \\
\cline { 2 - 5 } & 20160617 & 98.36 & 96.99 & 98.19 \\
\cline { 2 - 5 } & 20160713 & 99.53 & 88.63 & 98.02 \\
\cline { 2 - 5 } & 20160722 & 97.34 & 96.00 & 97.10 \\
\cline { 2 - 5 } & 20160729 & 98.91 & 82.09 & 95.48 \\
\hline & Average & $\mathbf{8 8 . 0 3}$ & $\mathbf{9 4 . 9 2}$ & $\mathbf{9 0 . 0 3}$ \\
\hline
\end{tabular}

Classification Using a Small UAV Based on Superpixel Segmentation and Machine Learning" RSSJ Journal of The Remote Sensing Society of Japan, 36(2), 59-71 (2016).

[3] Kageyama Y.; Ito N.; Isizawa C.; Nishida M.; Kon T.; Watanabe S., "Algorithm for Estimating Ratio of Harmful Vegetation to Manage River Bank" IEICE Transactions on Information and Systems 95(4), 1056-1060, (2012).

[4] Kageyama Y.; Matsu Y.; Murakami T.; Isizawa C.; Nishida M.; Sugai A.; Saitoh S.; Iyogi Y.; Kon T., "Use of Texture Information to Classify Vegetation in River bank by SVM," IEEJ Transactions on Electronics Information and Systems (in Japanese), 135(3), 349-350 (2014).

[5] Takagi M.; Shimoda H., "Handbook of Image Analysis," University of Tokyo Press (2004).

[6] Abe S., "Introduction to Support Vector Machine for Pattern Recognition," Morikita Publishing (2011).

[7] Yoshino H.; Tsuchiya A.; Suga K., "The characteristics of Slope failure of embankments by overtopping" JSCE (1980)

[8] The Color Science Association, "Handbook of Color Science," University of Tokyo Press (2011).

[9] Andreas C. Muller.; Sarah Guido, "Introduction to Machine Learning with Python" O'Reilly Media (2016).

[10] Bernt S.; James C., "Object Recognition using Multidimensional Receptive Field Histograms,” European Conference on Computer Vision, 1, 610-619 (1996). 